\title{
ANASTOMOSIS BETWEEN THE ASCENDING AORTA THE MAIN PULMONARY ARTERY IN THE TETRALOGY OF FALLOT
}

\author{
BY \\ JAMES S. DAVIDSON \\ From the Royal Infirmary, Bradford
}

(RECEIVED FOR PUBLICATION JULY 11, 1955)

In certain circumstances, because of the relative position or inadequate size of the vessels available for systemic-pulmonary artery anastomosis, an operation either of the Blalock or the Potts type may be difficult or impossible. Barrett and Daley (1949) have recorded several cases where such technical difficulties existed.

Anastomosis between the ascending aorta and the main pulmonary artery would seem to offer an additional site for anastomosis in cases where the classical anastomoses are difficult.

\section{CAse Reports}

CASE 1.-A girl aged 9 years was seen at the Bradford Royal Infirmary in 1950 . At the age of 3 months a congenital heart lesion had been diagnosed when she was taken to a paediatric clinic because of cough. Cyanosis was first noted at the age of 16 months when the child began to walk. Thereafter she was noticed to squat and to be unduly breathless on exertion. She had recurrent fainting attacks.

In 1950 the child was stunted and markedly cyanosed while at rest in her wheel-chair. Her exercise tolerance varied from day to day. At her best she could walk 20 yards on the level. The fingers and toes were clubbed and arachnodactyly was present. The child had strabismus. There was kyphoscoliosis with convexity of the thoracic spine to the right and prominence of the right side of the chest posteriorly.

The left radial pulse was not palpable. The blood pressure in the right arm was $110 / 70 \mathrm{~mm}$. Hg. No record of the blood pressure could be obtained on the left arm. Both femoral pulses were easily palpable.

There was a systolic murmur, maximal to the left of the sternum in the third interspace. The E.C.G. showed right axis deviation. Haemoglobin was $124 \%$ (19.8 g.).

Angiocardiography showed the tetralogy of Fallot, with marked overriding of the aorta and a rightsided aortic arch. The arch was high. The descending aorta did not reach a position on the left of the midline until it had entered the abdomen. The intrathoracic portion of the left subclavian artery was not demonstrated. Late filling of a small axillary artery was seen to occur. There was no post-stenotic dilatation of the pulmonary artery.
The child spent the next four years at a special school. When seen again her exercise tolerance was the same as before. The cyanosis was slightly more marked. Her haemoglobin was $130 \%(20.8 \mathrm{~g}$.). The left radial pulse was palpable with difficulty. The blood pressure was not recordable in the left arm. In the right arm the blood pressure was $112 / 70 \mathrm{~mm}$. $\mathrm{Hg}$.

At operation on March 8, 1955 (Fig. 1), an incision was made on the anterior aspect of the chest from just to the right of the midline towards the left axilla. The third left intercostal cartilage was resected, together with a portion of the third left rib. The sternum was divided transversely at the level of the third cartilage and the second right intercostal space was opened for a distance of 1 in. Both pleural cavities were opened. The ascending aorta was followed upwards as far as it was accessible. Its first branch was not encountered. The left subclavian artery could not be found. The pericardium was incised anteriorly, and the intrapericardial portions of the aorta and the pulmonary artery exposed. The origins of these vessels appeared to be almost in the same coronal plane, presumably because of an overriding aorta. Dissection was carried out to separate the ascending aorta from the main pulmonary artery, with division of the anterior and posterior layers of the serous pericardial sheath investing these vessels. Intervening areolar tissue was also divided. This dissection was aided by passing a finger of the left hand into the transverse sinus. A tape was passed round $\frac{\text { o }}{3}$ the ascending aorta, which was then drawn gently $>$ forwards and to the right. This facilitated dissection between the aorta and the pulmonary artery near their origins, and also the dissection between the aorta and of the anterior aspect of the right pulmonary artery. N When the ascending aorta had been separated from $N$ the main pulmonary artery and its right branch, it $\omega$ was possible to apply a Potts-Smith clamp to it so? as to isolate a portion on its left side. The main 0 pulmonary artery was isolated completely with the intention of applying a Potts-Smith clamp to it. It $\stackrel{\odot}{+}$ was found, however, that the main pulmonary artery 0 was too short for the application of the available 0 clamp. The ligamentum arteriosum, which passed $\overparen{D}$ posteriorly to the left of the trachea, was divided, $\frac{?}{\mathbb{Q}}$ and the end of it attached to the pulmonary artery was used to pull the pulmonary artery forwards so 


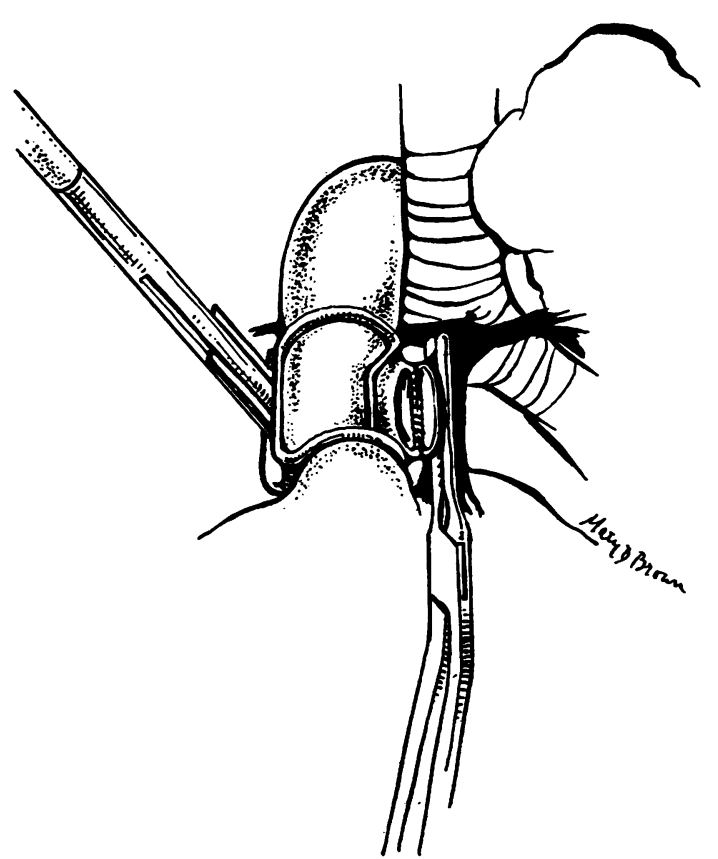

FIG. 1.-A drawing of the operation.

that a Potts ductus clamp could be applied longitudinally to the front of the main pulmonary artery so as to isolate a portion of the artery on its anterior aspect. The Potts-Smith clamp was then screwed home on the aorta. An anastomosis about $4 \mathrm{~mm}$. in length was made between the two vessels. The clamp was removed from the pulmonary artery and then the aortic clamp was released. To begin with, no thrill was palpable. Suddenly a good thrill was felt, and the pulmonary artery increased in size and tension. Apparently the clamp applied to the main pulmonary artery had caused the intimal surfaces of the vessel to adhere temporarily at the site of pressure from the clamp.

The post-operative course was uneventful. On the day after operation the cyanosis was less. A systolic murmur was audible over the praecordium, together with a continuous murmur best heard over the manubrium sterni and in the second right intercostal space. Two weeks after operation the haemoglobin was $98 \%$ $(15.7$ g.) and the blood pressure in the right arm was $110 / 50 \mathrm{~mm}$. $\mathrm{Hg}$. The child was discharged from hospital 22 days after operation.

Five weeks later she came to hospital by bus. She walked a quarter of a mile uphill to get the bus, and a further quarter of a mile to the hospital. She was then able to mount a flight of stairs. She had not squatted since the operation. Since beginning to walk about after the operation she had complained of aching pains in her back and in her feet. She was moderately cyanosed on exertion. At rest she was pink. Blood pressure was $110 / 70 \mathrm{~mm}$. $\mathrm{Hg}$, haemoglobin $102 \%$ (16.3 g.).
Three months after operation exercise tolerance was increasing still further. The continuous murmur was louder than before. There was no thrill. Radiographs showed evidence of only slight cardiac enlargement as compared with the pre-operative films.

CASE 2.-An undernourished baby boy aged 11 months had been diagnosed as a case of cyanotic congenital heart disease at the age of 9 weeks. The baby had repeated attacks in which he became deeply cyanosed and lost consciousness. During some of these attacks death appeared to be imminent. The fingers and toes were clubbed. A systolic murmur was heard to the left of the sternum. Haemoglobin was $110 \%(17.6 \mathrm{~g}$.). Angiocardiography showed the tetralogy of Fallot, with a right-sided aortic arch and an anomalous vessel in the upper part of the left side of the chest.

While in hospital the baby had several episodes of loss of consciousness during which his condition became desperate.

The chest was opened through a left postero-lateral incision with the intention of performing Blalock's operation. The left pulmonary artery was small. The left subclavian artery was found passing upwards in the posterior mediastinum. At the thoracic inlet it gave off a large branch which passed forwards and then turned back on itself in a hair-pin manner and proceeded downwards towards the hilum of the left lung. Just above the hilum it divided into two branches which entered the hilum anterior to the left pulmonary artery. The left internal mammary artery was given off as a small branch of this vessel, taking origin from the convexity of its acute bend. The left pulmonary artery appeared to be too small for the purpose of anastomosis.

An incision was then made anteriorly as in the previous case. Both pleural cavities were opened. As in Case 1 , the origins of the aorta and the pulmonary artery were more nearly in the same coronal plane than is the case in the normal heart. As in the previous case, the pulmonary artery and its right branch were freed from the ascending aorta. A Potts ductus clamp was applied longitudinally to isolate part of the main pulmonary artery on its anterior aspect. A Potts-Smith clamp was applied to the ascending aorta so as to isolate a segment on the left side of the vessel. When the incision in the aorta was about to be made it was noticed that the heart rate was becoming progressively slower. The aortic clamp was released and the heart's action rapidly recovered. The clamp was then moved as far as possible along the aorta away from the heart and reapplied. The heart appeared to be beating satisfactorily. An incision was made in the aorta. At this point the heart beat again became very slow. The aortic incision was rapidly sutured and the aortic clamp released. Again recovery took place. A further attempt to apply the aortic clamp had similar results. The operation was abandoned. The baby died later on the same day.

At necropsy there was an infundibular stenosis and gross narrowing of the segment containing the pulmonary valve. Judging from the position of the suture 
line in the ascending aorta it seemed certain that the clamp had occluded the origin of the left coronary artery.

\section{Discussion}

Blalock and Taussig (1945), in their original article on the surgical treatment of malformations of the heart in which there is pulmonary stenosis or pulmonary atresia, mention the possibility of making an anastomosis between the main pulmonary artery and the ascending aorta. At that time a suitable instrument was not available for partial occlusion of the aorta, and anastomosis at this site was not carried out in man (Blalock, personal communication). With the introduction of the PottsSmith clamp, partial occlusion of the aorta became possible. The operation described here is merely the operation of Potts, Smith, and Gibson (1946) carried out at a different site. As the presence of a high interventricular septal defect and an overriding aorta provide an outlet for the blood flow from the right ventricle, partial occlusion of the main pulmonary artery causes little apparent cardiac embarrassment.

Scott and Sabiston (1953) record the creation of an aortico-pulmonary anastomosis in dogs. Their object in the creation of this opening was to gain experience in the closure of congenital aorticopulmonary fistula in man.

In most cases the main pulmonary artery is larger than either of its branches. Where the branch pulmonary arteries are inadequate for anastomosis, the main pulmonary artery may be large enough to allow of a satisfactory anastomosis being made. The ascending aorta and the main pulmonary artery are in apposition, so that the question of there being too great a distance between the vessels selected for anastomosis does not arise. With a marked degree of overriding of the aorta, the initial course of the aorta and the main pulmonary artery may be almost parallel, though the pulmonary artery then passes posteriorly, crossing the left side of the ascending aorta obliquely.

In Case 2 it might have been best to anastomose the left subclavian artery to the main pulmonary artery, and to ignore the large branch of the left subclavian artery passing into the hilum of the left lung. The attempt at anastomosis of the main pulmonary artery and the ascending aorta failed for technical reasons. Their size and anatomical position were such that an anastomosis should have been possible. It is not known whether the cardiac failure which occurred after applying the aortic clamp was the result of excessive occlusion of the aorta, or of occlusion of the left coronary artery, or of both these factors. At necropsy there seemed little doubt that the left coronary artery had been obstructed. In Case 1 the ascending aorta was longer and of larger calibre. The application of the Potts-Smith clamp to the ascending aorta caused no apparent cardiac embarrassment. In small babies the ascending aorta is short and the aorta soon arches posteriorly. Attempts are being $\vec{\circ}$ made to modify the existing types of clamp for use on this vessel. In Case 2 the available PottsSmith clamp required too great a length of the ascending aorta for its application. Also this clamp is perhaps not ideal for application to an arching vessel.

In the successful case the anastomosis was not difficult. The result of the operation has, after three months, been comparable with that of the standard Blalock and Potts procedures. It is suggested that in certain circumstances, which, however, must be rare, anastomosis at this site may be useful. In particular, it is suggested that such an anastomosis may have an application where surgery has to be attempted in young babies in whom the more peripheral vessels are small.

Certain disadvantages are apparent. With the clamp; available at present, there would appear to be a danger of excessive occlusion of the ascending aorta so that heart failure occurs as a result of obstruction of the outflow of the ventricles. Secondly, there is a danger of obstruction of the left coronary artery. As in other arterial anastomoses of this nature, there is a danger of the creation of too large an opening between the systemic and the pulmonary artery. This danger is not peculiar to anastomosis at the present site. It may be that the haemodynamic effect of this anastomosis differs from that of the Blalock or the Potts procedures. Finally, technical failure of an anastomosis at this site may have more disastrous immediate results if haemorrhage is difficult to control.

SUMMARY
A case of Fallot's tetralogy is described in which
anastomosis was made between the ascending
orta and the main pulmonary artery.
Possible indications for such an operation are
mentioned.

Apparent dangers and disadvantages of the operation are discussed and illustrated by the 0 description of an unsuccessful case.

I am indebted to Miss M. D. Brown for the drawing.

\section{REFERENCES}

Barrett, N. R., and Daley, R. (1949). Brit. med. J., 1, 699.

Blalock, A. (1955). Personal communication.

Potts, W. J., Smith, S., and Gibson, S. (1946). Ibid., 132, 627

Scott, H. W., and Sabiston. D. C. (1953). J. thorac. Surg., 25, 26.

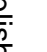

